Bundesgesundheitsbl 2012 · 55:1241-1242

DOI 10.1007/s00103-012-1571-7

Online publiziert: 22. September 2012

(c) Springer-Verlag 2012
M. Mielke ${ }^{1}$ N N.-O. Hübner ${ }^{2}$

${ }^{1}$ Leitung Abteilung 1, Robert Koch-Institut, Berlin

${ }^{2}$ Leitung FG 14, Robert Koch-Institut, Berlin

\title{
Aus der Arbeit der Kommission für Krankenhaushygiene und Infektionsprävention
}

Seit der Aufdeckung der Ursachen von Infektionskrankheiten, an der Robert Koch maßgeblich beteiligt war, konnten enorme Fortschritte zur Vermeidung und Behandlung übertragbarer Erkrankungen erzielt werden. Infektionskrankheiten haben dadurch viel von ihrem Schrecken verloren. Es wundert daher nicht, dass Berichte über Infektionen, die im Zusammenhang mit medizinischen Maßnahmen auftreten (nosokomiale Infektionen), großes öffentliches Interesse erregen. Dies gilt umso mehr, wenn diese Infektionen durch schwer behandelbare Erreger ausgelöst werden und nicht nur in Einzelfällen, sondern als Häufungen und mit Todesfolge in Risikobereichen von Krankenhäusern auftreten.

Eine ähnliche Aufmerksamkeit finden auch Berichte über Mängel bei der Aufbereitung von chirurgischen Instrumenten. Auch hier wird angenommen, dass seit den bahnbrechenden Arbeiten Robert Kochs und anderer zur Desinfektion und Sterilisation die Probleme eigentlich gelöst seien. Nicht zuletzt die Berichte über Infektionen bzw. Ausbrüche mit ESBL-bildenden Darmbakterien auf neonatologischen Intensivstationen sowie Beanstandungen bei der Aufbereitung von Medizinprodukten haben daher zu einer Novellierung des Infektionsschutzgesetzes und anderer Gesetze beigetragen, die am 3.08.2011 im Bundesgesetzblatt veröffentlicht wurde. Zentraler Bestandteil sind Änderungen, die einen verbesserten Schutz vor Infektionen mit schwierig zu behandelnden Erregern zum Ziel haben. Dazu gehören Änderun- gen, die die Weitergabe von Meldungen über das gehäufte Auftreten nosokomialer Infektionen vom Gesundheitsamt an das Robert Koch-Institut betreffen, die Aufgaben und Verantwortlichkeiten der Leiter von medizinischen Einrichtungen im Bereich der Infektionsprävention konkretisieren und Aspekte des Gebrauchs von Antibiotika (Antiinfektiva) einschließen. Der neue und umfangreichere $\$ 23$ IfSG nimmt hier eine zentrale Stellung ein. Für die Umsetzung wesentlich ist die Präsenz von Fachpersonal für die Gebiete Hygiene und Antibiotikatherapie in den jeweiligen Einrichtungen.

Aufgrund ihrer Häufigkeit und Bedeutung als Infektionserreger geben nach wie vor MRSA sowie wegen der zunehmenden Dynamik der Ausbreitung insbesondere gramnegative Bakterien wie E. coli, Klebsiella pneumoniae, Pseudomonas aeruginosa und Acinetobacter spp. mit Multiresistenzen Anlass zur Sorge. Die Kommission für Krankenhaushygiene und Infektionsprävention (KRINKO) hat zu diesem Thema im Verlauf des Jahres 2011 wichtige Mitteilungen erarbeitet ["Definition der Multiresistenz gegenüber Antibiotika bei gramnegativen Stäbchen im Hinblick auf Maßnahmen zur Vermeidung der Weiterverbreitung“" (EpiBull 36/2011); „Ergänzende Empfehlung (2011) zur ,Prävention nosokomialer Infektionen bei neonatologischen Intensivpflegepatienten mit einem Geburtsgewicht unter 1500 g' (2007)“ (EpiBull 2/2012)]. Diese Mitteilungen werden jetzt durch die „Hygienemaßnahmen bei Infektionen oder Besiedlung mit multi- resistenten grannegativen Stäbchen“ vervollständigt, die sich in diesem Heft finden.

Während es sich bei der Empfehlung zu gramnegativen Problemerregern um eine neue Empfehlung der Kommission handelt, stellen die „Anforderungen an die Hygiene bei der Aufbereitung von Medizinprodukten" eine Aktualisierung einer bestehenden Empfehlung aus dem Jahr 2001 dar.

Die sog. KRINKO-BfArM-Empfehlung bedurfte einer Aktualisierung, um weitere von der KRINKO und dem BfArM zwischenzeitlich veröffentlichte Empfehlungen und Kommentare zu speziellen Bereichen der Aufbereitung und andere aktuelle Veröffentlichungen - wie z. B. den Erfahrungsbericht zur Aufbereitung von Medizinprodukten in Deutschland des Bundesministeriums für Gesundheit aus dem Jahre 2008, europäische und nationale Standards/ Normen, Originalveröffentlichungen zu Aspekten der Aufbereitung sowie Leitlinien und Empfehlungen von Fachgesellschaften - zu berücksichtigen. In einer interdisziplinären Arbeitsgruppe wurde entsprechend dem in der KRINKO geübten Verfahren ein neuer Entwurf der KRINKO-BfArM-Empfehlung erstellt und nach Beratung in der Kommission das für Empfehlungen der KRINKO vorgesehene Anhörungsverfahren unter Beteiligung von Ländern und Verbänden durchgeführt. Die anschließende Beratung der eingegangenen Kommentare in der Kommission unter Beteiligung des BfArM führte schließlich zur Verab- 
schiedung der aktualisierten, hier vorliegenden Empfehlung.

Die zentrale Empfehlung „Anforderungen an die Hygiene bei der Aufbereitung von Medizinprodukten“ ist mit Rücksicht auf die erfolgreiche Anwendung in der Vergangenheit in ihrem prinzipiellen Aufbau größtenteils gleich geblieben und wurde jeweils an den entsprechenden Stellen aktualisiert bzw. um einzelne Aspekte (wie z. B. konkretisierende Angaben zu manuellen Schritten der Aufbereitung, Zwischenspülung, Prüfung der viruziden Wirksamkeit eines Desinfektionsmittels, Heißluftsterilisation, Rückverfolgbarkeit) ergänzt.

Neu sind insgesamt 8 dazugehörige Anlagen und weitere Anhänge zur Konkretisierung von einzelnen Aspekten der allgemeinen Empfehlung.

Die genannten Empfehlungen gelten grundsätzlich unabhängig vom Ort der Durchführung der Aufbereitung sowohl im ambulanten und stationären Sektor. Entscheidend für Art und Umfang der Maßnahmen sind die Komplexität des Medizinproduktes sowie die vorausgehende und die der Aufbereitung folgende Anwendung.

Die beiden Empfehlungen stellen in gewisser Hinsicht zwei Pole der Beherrschbarkeit von Risiken dar. Während die Aufbereitung von Medizinprodukten durch Standardarbeitsanweisungen und technische Prozesse in hohem Maße reproduzierbar und durch Validierung hinsichtlich des angestrebten Ergebnisses zielführend ist, unterliegt die Beherrschung von Infektionsrisiken durch mehrfachresistente Darmbakterien einer Vielzahl nur zum Teil beeinflussbarer Faktoren. Dementsprechend unterschiedlich sind der Umgang mit Evidenzkategorien und der Effekt qualitätssichernder Maßnahmen in den beiden Bereichen.

Die Kommission und insbesondere die vorbereitenden Arbeitsgruppen haben sich die Arbeit nicht leicht gemacht, und es ist hier die Stelle, den Beteiligten und bei der jeweiligen Empfehlung Genannten dafür ausdrücklichen Dank $\mathrm{zu}$ äußern. Es ist Empfehlungen dieser Art eigen, dass ihre Anwendung zu Rückfragen führt. Wir bitten daher alle Anwender der Empfehlung, ihre Fra- gen oder Anregungen jeweils möglichst unter Nennung der konkreten Erfahrungen bzw. von entsprechenden Literaturquellen mitzuteilen (Infektionshygiene@ rki.de). So kann der Prozess fortlaufender Verbesserung gemeinsam gelingen.

Ihre

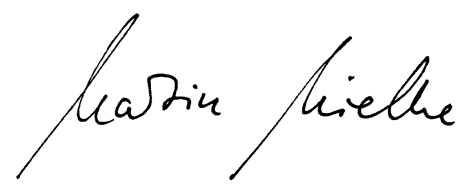

Martin Mielke

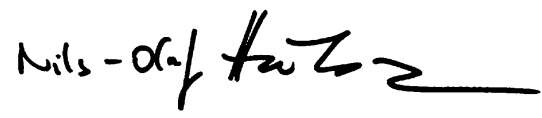

Nils-Olaf Hübner

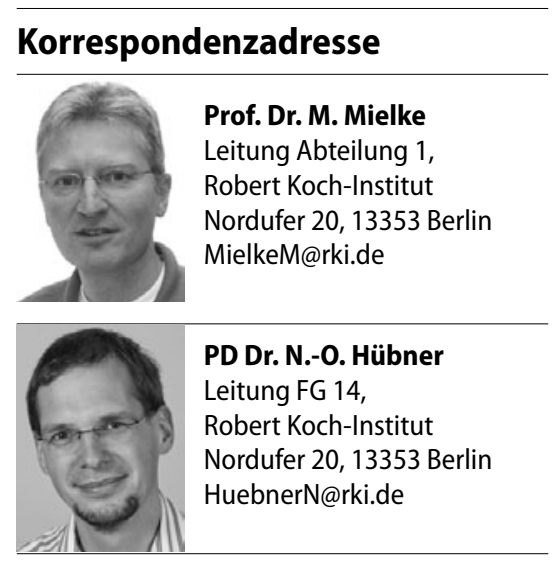

